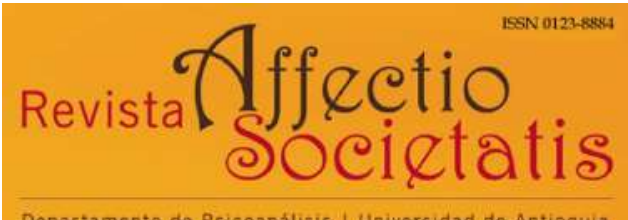

Departamento de Psicoanálisis | Universidad de Antioquia

Revista Affectio Societatis

Departamento de Psicoanálisis

Universidad de Antioquia

revistaaffectiosocietatis@udea.edu.co

ISSN (versión electrónica): 0123-8884

Colombia

Milany Andrea Gomez, Maria Elena Restrepo

Psicoanálisis y filosofía política: una diada óptima para la explicación del comportamiento electoral colombiano

Revista Affectio Societatis, Vol. 18, N. ${ }^{\circ}$ 34, enero-junio de 2021

Art. \# 4 (pp. 1-26)

Departamento de Psicoanálisis, Universidad de Antioquia

Medellín, Colombia 
ARTÍCULO DE INVESTIGACIÓN

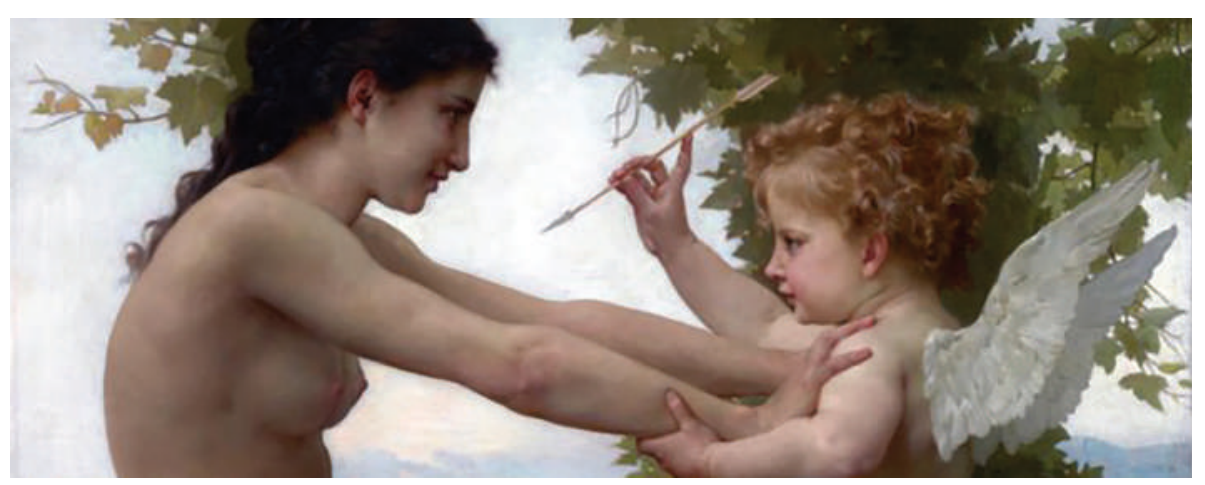




\title{
PSICOANÁLISIS Y FILOSOFÍA POLÍTICA: UNA DIADA ÓPTIMA PARA LA EXPLICACIÓN DEL COMPORTAMIENTO ELECTORAL COLOMBIANO'
}

\author{
Millany Andrea Gómez Betancur² \\ Universidad Católica de Oriente, Colombia \\ mgomez@uco.edu.co \\ https://orcid.org/0000-0001-9362-3896
}

María Elena Restrepo Vélez ${ }^{3}$

Universidad Católica de Oriente, Colombia

nenarestrepovelez@gmail.com

https://orcid.org/0000-0003-3780-9383

DOI: 10.17533/udea.affs.v18n34a04

\section{Resumen}

El presente artículo es una aproximación al pensamiento del filósofo esloveno Slavoj Žižek, y a sus fundamentos lacanianos en torno a la con-

cepción del otro, y la interpretación de la forma en que la crisis migratoria venezolana de la actualidad influyó en el voto al candidato presidencial

1 El presente artículo es resultado de la investigación denominada "Análisis comparado de las principales corrientes del multiculturalismo contemporáneo (capitalismo, subjetividad y derechos humanos)" financiada por la Universidad Católica de Oriente y adscrita al grupo Humanitas del Departamento de Humanidades de la misma institución. Esta investigación fue finalizada en el año 2019.

2 Filósofa. Magíster en Relaciones Internacionales y candidata a doctora en Marketing Político, Actores e Instituciones en las Sociedades Contemporáneas. Coordinadora de la Maestría en Humanidades de la Universidad Católica de Oriente y docente y miembro del grupo de investigación Humanitas de la misma institución. Investigadora categorizada en el sistema de investigación colombiano Minciencias. Autora de diferentes artículos y capítulos de libros.

3 Magíster en Humanidades de la Universidad Católica de Oriente y co-investigadora grupo Humanitas. 
Iván Duque durante las elecciones colombianas del 2018. A su vez, es resultado de investigación del proyecto denominado "Un análisis comparado de las principales corrientes teóricas del multiculturalismo contemporáneo: capitalismo, subjetividad y de- rechos humanos", financiado por la Dirección de Investigación y Desarrollo de la Universidad Católica de Oriente.

Palabras clave: capitalismo, elecciones, Lacan, Žižek, Venezuela.

\section{PSYCHOANALYSIS AND POLITICAL PHILOSOPHY: AN OPTIMAL DYAD FOR EXPLAINING COLOMBIAN ELECTORAL BEHAVIOR}

\section{Abstract}

This paper is an approach to the thinking of the Slovenian philosopher Slavoj Žižek and his Lacanian foundations on the conception of the other. It is also an interpretation of how the current Venezuelan migratory crisis influenced the vote in favor of the presidential candidate Iván Duque during the 2018 Colombian elections. It is the result of the re- search project "A comparative analysis of the main theoretical currents of contemporary multiculturalism: capitalism, subjectivity, and human rights," financed by the Research and Development Office of the Universidad Católica de Oriente.

Keywords: capitalism, elections, Lacan, Žižek, Venezuela.

\section{PSYCHANALYSE ET PHILOSOPHIE POLITIQUE : UNE DYADE OPTIMALE POUR EXPLIQUER LE COMPORTEMENT ÉLECTORAL COLOMBIEN}

\section{Résumé}

Cet article propose un rapprochement entre la pensée du philosophe slovène Slavoj Žižek, et ses fondements lacaniens au sujet de la conception de l'autre, ainsi que l'interprétation de la manière dont la crise migratoire vénézuélienne actuelle a influencé le vote pour le candidat présidentiel Iván Duque lors des élections colombiennes en 2018. L'article est également le résultat d'un projet de recherche intitulé « Analyse com- 
parative des principaux courants théoriques du multiculturalisme contemporain : capitalisme, subjectivité et droits de l'homme », financé par la Direction de la Recherche et du Développement de l'Universidad Católica de Oriente.

Mots-clés : capitalisme, élections, Lacan, Žižek, Venezuela.

\section{PSICANÁLISE E FILOSOFIA POLIITICA: UMA DÍADE IDEAL PARA EXPLICAR O COMPORTAMENTO ELEITORAL COLOMBIANO}

\section{Resumo}

O presente artigo é uma abordagem ao pensamento do filósofo esloveno Slavoj Žižek e a seus fundamentos lacanianos em torno da concepção do outro, e a interpretação da forma como a atual crise migratória venezuelana influenciou o voto para o candidato presidencial Iván Duque durante as eleições colombianas de 2018. Por sua vez, é o resultado da pesquisa do projeto denomina- do "Uma análise comparativa das principais correntes teóricas do multiculturalismo contemporâneo: capitalismo, subjetividade e direitos humanos", financiado pela Diretoria de Pesquisa e Desenvolvimento da Universidad Católica de Oriente.

Palavras-chave: capitalismo, eleições, Lacan, Žižek, Venezuela. 


\section{Introducción}

Colombia ha sido un país caracterizado por una historia de grupos violentos y al margen de la ley. Desde la contienda entre liberales $\mathrm{y}$ conservadores a principios del siglo $\mathrm{xx}$, hasta los grupos de guerrillas y autodefensas gestados en los años 60, el país ha vivido una constante de momentos violentos con graves repercusiones para sus habitantes (Molano, 2016).

A pesar de lo anterior, durante el gobierno de Juan Manuel Santos, a sus ciudadanos se les dio la oportunidad de $\operatorname{votar}^{4}(2016)$ por acabar el conflicto y controlar los niveles de corrupción ${ }^{5}$ (2018), pero increíblemente el resultado fue un voto negativo a estas propuestas. ¿Por qué lo hicieron? ¿Quieren los colombianos mantener un Estado en guerra y con altos índices de corrupción? Esta no parece una elección que busque la mejora de las condiciones de cada ciudadano; entonces ¿por qué votaron de dicha manera? Pues bien, una de las variables que debe tenerse presente a la hora de analizar el comportamiento de los colombianos al momento de votar el referendo por la paz en el año 2016 y la elección del presidente de derecha, Iván Duque, en el año 2018, es que ambos sucesos estuvieron traspasados por un discurso de incitación al temor y al odio por la elección de políticas o candidatos que, de seguir con el acuerdo de paz, convertirían a Colombia en Venezuela, llevando al país a la crisis que el vecino

4 El plebiscito sobre los acuerdos de paz de Colombia de 2016 fue el mecanismo de refrendación para aprobar los acuerdos entre el Gobierno de Colombia y la guerrilla de las Fuerzas Armadas Revolucionarias de Colombia (FARC). Este fue rechazado por la población colombiana.

5 La consulta popular anticorrupción fue un proceso electoral que se realizó el domingo 26 de agosto de 2018 en Colombia. La papeleta de votación constó de siete preguntas para que los votantes pudieran aprobar o rechazar ( $\mathrm{Sí}$ o $\mathrm{No}$ ). La realización de la consulta fue aprobada por 84 votos a favor y 0 en contra el 5 de junio de 2018 en la plenaria del Senado de la República. Para que fueran aprobados los mandatos, era necesario obtener al menos 12'140.342 de votos (33,3 \%) y que el Sí obtuviera más del 50 \% de los votos. De haber sido aprobada se habría debido tramitar dicha norma por el Congreso de la República; sin embargo, la alta abstención electoral durante la jornada impidió que se alcanzara el umbral $\mathrm{y}$, por tanto, no fue aprobada. 
país afronta y generando las dinámicas de migración y pobreza que a diario se visibilizan en Colombia con el éxodo de venezolanos al país desde aproximadamente el año 2013. Por medio de Fake News, vallas publicitarias, mensajes en redes sociales, y discursos ante los medios, se creó lo que se ha denominado un discurso "castrochavista" a partir del cual se asimilaron los asuntos tendientes a la paz y el proceso de paz, con políticas de izquierda que decantarían necesariamente en una "venezolanización" de Colombia.

En este orden, si se toma como base la teoría de la affective intelligence (Marcus, et al., 2000) según la cual existen unas lógicas valenciales tanto positivas como negativas que hacen posible que la respuesta emocional del elector ser relacione con lo que le hace sentir el candidato. En el caso, desde la campaña presidencial de Iván Duque se prefirió la generación de temor y angustia hacia al candidato oponente tras la rememoración de hechos comunes de violencia en el territorio, con ello se logró un voto habitual que no necesitó la activación de un "sistema de vigilancia", o sea racional, que llevara al elector a una búsqueda de información sobre el candidato, sus propuestas, la problemática subyacente y la toma de decisión. Por ello se generó fue un sentimiento de empatía hacia el candidato de oposición a las políticas del expresidente Juan Manuel Santos, y el voto fue emocional.

Dado lo anterior, este artículo sostiene que si bien hubo un sentimiento de angustia que dentro de la lógica valencia llegó a generar una visión negativa de los planes y proyectos de paz o de la posible elección del candidato pro paz -Gustavo Petro-, no implicó esto un acto de racionalidad ni de búsqueda de información de sus propuestas. Lo que se puede evidenciar es que hubo una movilización de emociones que no se circunscriben meramente al temor o la angustia. Tomando como base el planteamiento del Slavoj Žižek sobre el multiculturalismo (2008), el cual parte de una base lacaniana, se sostendrá que dicha elección estuvo traspasada por el odio hacia el otro, la falacia multicultural del capital y un discurso que hacía posible ese devenir de lo Real a nivel político.

Así, para el desarrollo de este artículo: en un primer momento, 1) se expondrá su concepción filosófica sobre el multiculturalismo, 
explicitando a.) las nociones de sujeto, síntoma y ontología de la incompletitud, b) El sentido de lo real, lo simbólico y lo imaginario, y c) la noción de goce. De esta manera, con base en las tres reflexiones anteriores, en el numeral 2 expondrá por qué y de qué manera desde la interpretación del multiculturalismo planteado por Žižek se puede analizar el comportamiento electoral de esta contienda política en Colombia

\section{Sobre las nociones de sujeto, síntoma y ontología de la incompletud en Slavoj Žižek}

Para iniciar es primordial destacar que en Žižek la noción de sujeto se aparta del enfoque historicista ${ }^{6}$. Al revisar algunas de sus obras más importantes se descubre que su noción de sujeto se estructura a partir de la interpretación del idealismo alemán, incorporándole una actualización desde el psicoanálisis de Jacques Lacan. Así, comenzando por Descartes, realiza su primer acercamiento a la noción de sujeto más allá de una comprensión tradicional como ser racional y con capacidad completa de entendimiento. En su reinterpretación, Žižek (2003a) percibirá al sujeto cartesiano como sujeto dividido, que no posee atributos y que es sujeto de enunciación, por oposición al sujeto enunciado que posee unos atributos. Según Descartes, el sujeto es una cosa pensante (res cogitans), y en tal sentido es una cosa que duda. Es así como el sujeto no puede dejar de pensar sin dejar de ser, puesto que es una cosa que piensa, y por ello existe, $y$, en consecuencia, si no piensa tampoco existe. Ahora bien, según Žižek (2003b), el sujeto de Descartes no abandona la duda mediante la certeza, sino que, por el contrario, es un sujeto que tiene la certeza de la duda, que no es posible satisfacer en su plenitud -y por tanto no puede alcanzar el objeto del deseo-, configurando así para Žižek la comprensión de un sujeto que no puede llenar su vacío porque desborda lo histórico, porque es

6 La ontología de Foucault es una ontología historicista que se puede evidenciar en su ejercicio de leer un tiempo presente sin invocar un ente fundador. De esta manera, el sujeto es el producto de los modos de subjetivación que tienen carácter histórico. Para ampliar esta concepción, revisar Castro (2004, págs. 253-260). 
anterior a todo proceso de subjetivación": "Lo que caracterizaría entonces al sujeto cartesiano no es la transparencia y la soberanía, sino la hendidura y la división" (Castro-Gómez, 2004, págs. 23-24).

En este mismo sentido, Žižek (1998) reconoce en Kant el filósofo que hace un proceso de concreción de la noción de sujeto cartesiano, la cual inicia con la constitución trascendental de la realidad. Para él, es el sujeto el que construye el objeto, y cualquier experiencia o concreción de objetos en el mundo son deducidos gracias al sujeto. Cabe destacar aquí dos elementos del pensamiento kantiano: en primer lugar, el interés en no describir la estructura del mundo, sino por el contrario la forma de representación de esta estructura. En segundo lugar, Kant plantea imposible el encuentro con la verdad, ya que se debe buscar la percepción de esta a través de la ilusión. El ser humano está imposibilitado para conocer el mundo y su único camino es lograr su representación: “Aunque la cosa ${ }^{8}$-en-sí existe, de ella no podemos saber ni experimentar nada; tan sólo podemos conocer el proceso epistémico que se desencadena una vez producida la intuición sensible" (Castro-Gómez, 2015, pág. 31); y es allí en donde reside el vacío constitutivo del sujeto, en la necesidad de la representación $\mathrm{y}$, a su vez, en la forma incompleta de esa representación, por ello se

7 Para Santiago Castro-Gómez, el retorno de Žižek a Descartes tiene el objetivo de restablecer la conexión existente entre sujeto y verdad que se rompió por la filosofía del siglo xx conduciendo a un relativismo cultural incapaz de enfrentar al universalismo del capital.

8 La Cosa abre al pensamiento una dimensión no esperada y profunda de un goce del ser que es anterior a la existencia y que tiene un efecto retroactivo del lenguaje que, al ubicarse más allá de la cosa misma -del referente según los lingüistascrea la intuición de un más acá. La Cosa resulta así un efecto del lenguaje que introduce la falta y que separa de ella. La Cosa es irrepresentable, un escenario vacío en donde los objetos que pretenden sustituirla y poblar su espacio solo alcanzan un estatuto espectral, imaginario. Sugiere así la distinción entre la Cosa y los objetos indicando que cuando la Cosa falta es que los objetos del mundo aparecen y se multiplican, es el momento en que los hablantes, por medio del lenguaje, se dan un mundo y entran en el mercado del goce con el Otro. De esta manera los objetos son derivados de la pérdida, se constituyen en sus sucedáneos, en sus representantes fantasmáticos: “Del Goce, del Gran Goce inicial y mítico, a los gocecitos, a las minúsculas @ de los objetos que causan el deseo y lo vectorizan" (Castro-Gómez, 2015, págs. 105-106). 
afirma que el sujeto necesita dicha ilusión. Ello significa para Žižek que la epistemología de Kant muestra también una falla en el sujeto que es leída como una ontología incompleta.

Ahora bien, en Hegel encuentra la noción de sujeto concreto que tiene un origen histórico y que tiene la característica de proyectar su patología en el objeto. (Rodríguez de Dios, 2008) Si para Kant el sujeto es incompleto, para Hegel el mundo es incompleto y en él radica la inevitabilidad del antagonismo como la posibilidad que tiene el sujeto de ser. Esa negatividad que no puede ser superada se expresa en la división del sujeto y del ser (la sustancia), por lo cual es posible indicar que están intrínsecamente divididos y que, por tanto, no tienen posibilidad de una identidad plena y completa, lo que para Žižek significa que el antagonismo es una barrera que imposibilita la realización plena del sujeto (2003c). Žižek "insistirá en que tanto el sujeto como la sustancia se hallan atravesados por la contradicción. No existe por tanto ninguna reconciliación que cierre la grieta ontológica del ser. No hay salida posible a la tragedia del sujeto" (Castro-Gómez, 2015, pág. 48).

Al sumar a estas visiones del sujeto desde la óptica de Descartes, Kant y Hegel, su comprensión lacaniana del mismo, Žižek concluye que el sujeto está atravesado por una negatividad que le impide una identidad plena y completa. El sujeto es entonteces una representación que se logra a través de un significante: "El significante es lo que representa un sujeto para otro significante" (Chaumon, 2005), lo que está representado y es al mismo tiempo lo que vincula a un significante con otro. "Qué representa un significante? Un sujeto. ¿Para quién? (es decir, ¿Al lado de quién?) Para otro significante" (Chaumon, 2005, pág. 31). En el ámbito de la cura psicoanalítica lacaniana lo que circulan son significantes que remiten unos a otros de manera infinita, no buscando representar las cosas sino la presencia del sujeto, pues el sujeto no es evocado por sí mismo sino por otros significantes.

Como se puede apreciar, el sujeto está en constante búsqueda de significación, y lo hace a través del otro como estructura supuestamente significada, aunque en realidad tampoco este lo está. Es así como se estructura el sujeto y su realidad para Lacan, ejemplificada 
en lo que él denominó el nudo borromeo, una conformación de 3 registros con una posición equivalente: lo Real (R), lo Simbólico (S) y lo Imaginario (I), que precisa de los tres para su sostenimiento, y que se explicarán más adelante (Lacan, 1957 ) En términos de Lacan, la representación simbólica siempre deforma al sujeto puesto que es un desplazamiento, un fracaso, ya que el sujeto no logra hallar un significante que sea el suyo, que exprese de manera precisa lo que realmente pretendía decir (Žižek, 2003, pág. 227).

Ahora bien, entendido el sujeto como un vacío es necesario avanzar ahora hacia el entendimiento de la noción de síntoma como aquello que representa ese vacío, esa carencia ontológica. Para Lacan el síntoma como algo real va más allá de una codificación simbólica (1987); nada existe, pues no existe el mundo, no existe el lenguaje ni existe el sujeto y, por oposición, lo que sí existe y que sostiene la congruencia de todos los fenómenos es el síntoma. A su vez, el síntoma está relacionado con su formación patológica que, desde el punto de vista del psicoanálisis, persiste más allá de su interpretación y hace posible afirmar que llega más allá de la fantasía. Frente a esta formación Lacan propone el concepto de sinthome como una formación significante llena de goce, de jouis-sense, sentido de goce. El sinthome es, entonces, "nuestra única sustancia y soporte positivo de nuestro ser" (2006/1975-1976), que garantiza la congruencia del sujeto evitando que caiga en la locura; por ello para Lacan el fin último del proceso psicoanalítico es la identificación con el síntoma, "el análisis llega a su fin cuando el paciente es capaz de reconocer, en lo Real de su síntoma, el único soporte de su vida (...) tú, el sujeto, te has de identificar con el lugar en el que tu síntoma ya estaba; en su particularidad patológica" (Žižek, 2003, pág. 110).

Ahora bien, tras las nociones de sujeto y síntoma en Žižek es importante precisar la noción de ontología de la incompletud, y para ello cabe citar a Castro-Gómez (2015, pág. 33), quien afirma que para Žižek el sujeto debe identificarse con el acto primordial y absoluto del creador y así poder actuar libremente, es decir que debe elegirse a sí mismo sin recurrir a una razón dada por el mundo de lo simbólico: "El acto libre demanda una negación radical de todas las razones, una ruptura con la cadena de la causalidad de lo simbólico en la cual el 
sujeto existe. No hay libertad sin este acto de ruptura radical con el mundo en el cual ocupamos una posición de sujeto" (Castro-Gómez, 2015, pág. 39). Este acto, no obstante, es traumático.

\section{Las nociones sobre lo Real, lo Simbólico y lo Imaginario en Slavoj Žižek}

Para Lacan el inconsciente está estructurado como un lenguaje que posee su propia gramática -pues el inconsciente habla- y su propia lógica -pues el inconsciente piensa-. Lo que en el inconsciente reside es "una verdad insoportable con la que tengo que aprender a vivir" (Žižek, 2008, pág. 13). Para Lacan (1964), el psicoanálisis confronta al sujeto con la dimensión radical de la existencia humana y permite explicar cómo la realidad se constituye, es por ello que el propósito del tratamiento psicoanalítico es lograr que el individuo se confronte con las coordenadas de su deseo.

Así, en el psicoanálisis lacaniano, tres niveles aparecen superpuestos de manera parcial uno sobre otro: lo Simbólico, lo Imaginario y lo Real. En este orden, para la comprensión de lo simbólico, es primordial entender que su categoría central es la falta. Según lacan sólo puede haber falta si existe un sistema simbólico mínimo que permita inscribirla como tal, puesto que para pensar una cosa como faltante es necesario poder designar su ausencia. Lo Simbólico en la formación del sujeto corresponde entonces a la fase edípica, dentro del psicoanálisis freudiano, etapa en donde el individuo introyecta la normativa social entendida como conciencia moral. No obstante, en Lacan (1960) lo Simbólico es el mundo de las palabras que dan lugar al mundo de las cosas, es al mismo tiempo una conciencia que se representa a sí misma de modo absoluto, lo simbólico es para Lacan el lugar del código.

De esta manera, la expresión lacaniana "Gran Otro" opera en este nivel Simbólico que se puede definir como la constitución no escrita de la sociedad o, como el mismo Slavoj Žižek específica, la segunda naturaleza de todo ser hablante: "está ahí, dirigiendo y controlan- 
do mis actos; es el agua donde nado, en última instancia inaccesible -nunca puedo ponerlo en frente de mí y aprehenderlo-. Es como si nosotros, sujetos del lenguaje, habláramos e interactuáramos como marionetas, con nuestras palabras y gestos dictados por un poder omnipresente y anónimo" (Žižek, 2008b, pág. 18).

Así se entiende que el orden de lo Simbólico es el conjunto de reglas a las que el sujeto se subordina para interactuar con otros y que constituyen los parámetros para medirse. Ese conjunto de reglas está conformado básicamente por dos grupos: en primer lugar, las reglas gramaticales que se adoptan y se utilizan de manera automática para garantizar la continuidad del discurso; en segundo lugar, las reglas asociadas con el medio cultural que es comúnmente compartido con otros sujetos y que facilita el entendimiento entre los interlocutores. En este ámbito, el gran Otro de Lacan opera como "el Dios que vigila desde el más allá, a mí y a cualquier persona existente, o la causa que me compromete (libertad, comunismo, nación), por lo que estoy dispuesto a dar la vida. Mientras hablo, nunca soy un pequeño otro (individual) que interactúa con otros pequeños otros; el gran Otro siempre está ahí" (Žižek, 2008, pág. 19).

Lo que deseo está predeterminado por el gran Otro, el espacio simbólico que habito (...) el sujeto sólo desea en la medida en que percibe al Otro como deseante, como sede de un deseo indescifrable -como si un deseo opaco emanara de él o de ella-. No sólo el otro se dirige a mí con un deseo enigmático, también me confronta con el hecho de que yo mismo no sé qué es lo que realmente deseo, con el enigma de mi propio deseo (...) esta dimensión insondable de lo que es otro ser humano -el profundo abismo de una personalidad ajena, su absoluto hermetismo- encuentra expresión por primera vez en el judaísmo, con su imperativo de amar al prójimo como a uno mismo. (Žižek S, 2008, pág. 50).

El orden Simbólico dotado de reglas, pautas de actuación y obligaciones tiene como función fundamental que los individuos coexistan de manera mínimamente tolerable con otros individuos, y esto gracias a que un tercero se interpone entre unos y otros mediando para garantizar relaciones tolerablemente armónicas, evitando que la monstruosidad latente del ser humano salga a flote. 
Ahora bien, en segundo lugar está lo Imaginario, que desde Lacan corresponde a la fase pre-edípica de la formación del sujeto, en donde se forma un yo caracterizado por una imagen unificada de sí mismo, que establece una imagen ideal: "Es fuera de sí donde el sujeto capta su propia forma (...) la contemplación de la imagen de sí es a la vez reunión gozosa y dolor de no poder estar jamás a la altura de esta forma perfecta" (Chaumon, 2005, pág. 45). La realidad es lo Imaginario o lo que, en otras palabras, puede definirse como aquello

que constituye para el Sujeto una totalidad de sentido sin fisuras, que le da una plenitud ante sí mismo y ante los otros o, para mayor precisión: la realidad es un cierto anudamiento de lo Imaginario y lo Simbólico que permite que la experiencia compartida de la realidad deje lugar para la singularidad de la imagen vuelta sobre sí misma. (Jameson, 1998, pág. 49).

Lo Imaginario resulta imprescindible para la "simbolicidad", y es así como el denominado anudamiento entre lo Imaginario y lo Simbólico constituye la realidad -que no lo Real, como se verá más adelante-. En esta línea discursiva se puede afirmar que lo Simbólico se ancla sobre lo Imaginario para cuestionar de manera inconsciente la plenitud, permitiéndole al sujeto confrontarse con su propia falta, con su vacío. Desde lo Simbólico se declara que hay algo en la realidad que, si bien es vital, no garantiza su completa adopción de sentido, o, parafraseando, muestra que su sistema de equivalencias denota una carencia en el sentido que impide su articulación plena.

Finalmente está lo Real, este inicia en su diferenciación de realidad y es en esta diferenciación que se ajusta a lo postulado por Lacan. Lo Real es lo exterior al sujeto, en Lacan es aquello que "permanece idéntico en todos los universos posibles (de observación) (...) la distorsión y/o la disimulación son reveladoras por sí mismas: lo que emerge a través de las distorsiones de la representación fiel de la realidad es lo real; es decir, el trauma alrededor del cual se estructura la realidad social" (Žižek, 2003, págs. 36-37).

Lo Real es insubstancial y no puede ser integrado en el registro de lo Simbólico, es considerado por Lacan como un "núcleo duro" que 
se resiste a la simbolización. Si bien lo Real no tiene una existencia en la realidad, por su causalidad estructural produce efectos en la realidad simbólica de los sujetos, lo que, parafraseando a Žižek, permite afirmar que la definición de lo Real es la de "una causa que en sí no existe, que está presente sólo en una serie de efectos, pero siempre de un modo tergiversado, desplazado" (Žižek, 2003, pág. 214). El estatuto de lo Real es, entonces, el estatuto de lo imposible. El sujeto es así una forma de responder desde lo Real a las preguntas del orden simbólico que están representadas en el gran Otro y, por ende, el sujeto, que representa el objeto esencial en sí mismo, es un objeto que no puede ser dominado ni objetivado, para lo cual Lacan ha propuesto el denominado objeto a entendido como el punto de lo Real en el sujeto que es imposible de ser simbolizado.

La noción lacaniana de que lo Real es imposible no significa que no se pueda hacer nada con lo Real. La apuesta o esperanza fundamental del psicoanálisis es que con lo simbólico se puede intervenir en lo Real. Lo que Lacan llama el sinthome (su versión del síntoma) es Real; un Real simbólico en el sentido de que estructura el goce. (Žižek, 2006, pág. 143).

\section{La noción de goce en Slavoj Žižek}

En primer lugar, el objeto se comprende a partir de la invención de Freud como "un objeto perdido", pues no hay objeto sino sobre un trasfondo de falta, frente a lo cual Lacan indicó que "el objeto siempre está perdido ya que el sujeto se lanza en su búsqueda a partir de una primera falta (...) es el objeto (en la medida en que falta) lo que pone en movimiento al sujeto, y no el sujeto quien, por su propia iniciativa, se lanzaría a su conquista" (Chaumon, 2005, pág. 53).

Desde la concepción de Lacan sobre lo simbólico se puede afirmar que es gracias a que el sujeto ocupa un lugar en un mundo atravesado por el lenguaje, que los objetos que lo ponen en movimiento son, en primer lugar, objetos inscriptos en el campo del Otro y por ello adquieren valor. Los objetos adquieren valor de intercambio gracias a 
una lógica de objeto dado o rechazado, y por lo tanto se constituyen, según Franck Chaumon, en signos de odio, amor o indiferencia. Desde esta perspectiva los verbos mediante los cuales el sujeto inquiere por el lugar que ocupa para el otro se relacionan con dar y rechazar (devolver) lo que se traduce en una demanda de amor. Sin embargo, también existe, más allá de la demanda, la falta como característica propia del objeto, que adquiere valor en el lazo con el Otro.

En este punto aparece el deseo, entendido como la pregunta por lo que el Otro quiere más allá de lo que sus palabras dicen. Esta imposibilidad de saber con certeza lo que el Otro desea es el enigma del deseo del Otro que es, finalmente, el que orienta la búsqueda del objeto de deseo. Se identifican entonces tres objetos que pueden sintetizarse así: el objeto de la necesidad, que es mera subsistencia; el objeto de la demanda, que es interés por saber si se es amado; y el objeto del deseo, para preguntar por lo que está en juego en la relación con el Otro.

Cabe en este punto de la reflexión preguntar ¿qué es lo que da estatuto al Otro? Y es ese deseo antes descrito que opera como un enigma el que le otorga su condición de Otro, pues es ese a quien no se conoce sobre el que se puede afirmar que hay en él algo que se nos escapa al conocimiento. Ahora bien, si el Otro desea un objeto es debido a la falta generada sin saber que conlleva esa falta. Adicionalmente el Otro está afectado por un inconsciente que hace que ese deseo por el que se interroga sea también inconsciente. Para Lacan, el objeto del deseo no tiene imagen puesto que no es en el espejo en donde se encuentra la comprensión del deseo del Otro. Aquí surge la noción de objeto a entendido como esa cosa que es inaprensible y que impulsa hacia adelante al sujeto para ir en su búsqueda. También existe otro nombre para ese objeto a y es el plus-de-goce, que, en palabras de Franck Chaumon, signa ese anudamiento entre la falta, el eros y la muerte que Freud había discernido en la pulsión (Chaumon, 2005, pág. 60).

Por otra parte, cabe referir la expresión goce, que equivale a jouissance, y que es un concepto esencial en el psicoanálisis lacaniano. La comprensión de su sentido debe establecerse a partir de la oposición entre goce y placer, puesto que el principio de placer supone una limitación al goce. El sujeto está permanentemente buscando transgredir 
esas limitaciones para ir más allá del principio de placer (expresión de origen freudiano) y, en esa búsqueda, lo que el sujeto obtiene es un placer paradójico, "placer en el dolor", que constituye la marca del goce, en otras palabras, el sujeto obtiene una satisfacción paradójica. La explicación para esta paradoja desde la mirada de Žižek es que el goce que el sujeto experimenta se basa en la transgresión, que es algo impuesto, ordenado, es un mandato del superyó que invita al goce: “Lo mismo exactamente cabe decir de la concepción del deseo que aparece en Lacan; por eso el goce no es algo natural en el sujeto, la realización de un potencial interior, sino el contenido de un imperativo superyoico traumático" (Žižek, 2003, pág. 19).

El goce, desde la mirada de Lacan, es un fenómeno y no un concepto que se sostiene en el imperativo del superyo; este actúa en su rol de conciencia moral y regula las interacciones de los sujetos bajo la norma y la convivencia social. Desde esta perspectiva, el sujeto siempre está deseando algo que nunca logra atender su demanda y de allí que su deseo se mantenga. (Žižek, 2001) En consecuencia, socialmente se acepta la existencia de la castración simbólica, es decir, aquella que ofrece una defensa contra la pulsión insaciable y nunca completamente satisfecha que de no ser castrada -regulada- llevaría al sujeto a la destrucción.

\section{Multiculturalismo, un escenario de racialización dentro del capitalismo: el caso castrochavismo en Colombia}

Los estudios sobre el comportamiento electoral han pasado por varias etapas en el desarrollo como disciplina. Como parte de las ciencias políticas estos han seguido su trasegar, y desde la escuela de la racional choice (Down, 1975), las teorías estructuralistas y posestructuralistas, hasta la psicología social de corte gestaltista, se han interesado por indagar los móviles que llevan a los ciudadanos a elegir a un determinado representante político. Ahora bien, utilizando generalmente las encuestas como un método que presenta mayor fiabilidad, se han tomado diferentes clivajes principalmente para analizar este fenómeno, confiando en que la respuesta que racionalmente da 
el elector es la que ha motivado su voto. No obstante, en los últimos años, en que las emociones han reaparecido en el escenario académico como condicionantes de actitudes y acciones, diversos ciencistas políticos han emprendido la tarea de medir estas a través de diferentes variables que incluyen sentimientos y emociones dentro de los estudios demoscópicos.

Es claro, muchas encuestas cumplen su papel de predictores y analistas poselectorales de la percepción en el escenario electoral, sin embargo, hay casos donde el escenario político es complejo y los estudios estadísticos de este tipo fallan a la hora de hacer su trabajo. Una de las razones que esgrimimos puede ser que, precisamente, se confía en que el sujeto que las responde está dando una respuesta emocional, cuando el acto hacerlo consciente limita las actitudes inconscientes y plenamente emocionales. Por ello, sin negar la importancia de las encuestas para el campo de los estudios electorales, se considera que la filosofía política tiene mucho que aportar, principalmente a partir de los estudios posmarxistas sobre la ideología y el comportamiento, de la mano del psicoanálisis. Con base en estos se puede analizar qué discursos movilizan a los ciudadanos a elegir, desde su constitución ontológica como sujetos, y no solamente desde su estructura histórica-cultural.

Para ello, entones, cabe recordar que en Žižek el sujeto se encuentra atravesado por una grieta ontológica que es constitutiva de su ser y para la que no existe salida posible. El sujeto se encuentra atado por la imposibilidad de encontrar un significante que exprese de manera precisa lo que realmente quiere o pretende decir y, en tal sentido, la representación simbólica a la que recurre es un desplazamiento que siempre lo deforma como sujeto. La alternativa frente a esta condición es el surgimiento del sinthome como soporte positivo que garantiza la congruencia del sujeto para evitar que caiga en la locura, y de esta manera el sujeto se constituye en una respuesta del núcleo traumático -de lo Real- a la pregunta del Otro.

En este orden, como lo refiere Žižek, en el mundo capitalista la democracia liberal representa la forma de simbolizar el deseo de los sujetos y garantizar el goce, de tal suerte que les permita mantener su fidelidad 
con el sistema capitalista. Es necesario precisar que se trata de un capitalismo global catalogado como tardío multiculturalista, pues está caracterizado por un síntoma que es el racismo contemporáneo. Es decir, según Žižek, la tolerancia del capitalismo liberal excusa al Otro folclórico, pero denuncia a cualquier Otro real por su fundamentalismo (Žižek, 2011b). Dicha tolerancia es en la actualidad la categoría ideológica dominante debido a que en la mayoría de las democracias en Occidente las injusticias sociales son asumidas como problemas de intolerancia y no como problemas de carácter económico que generan afectación a la sociedad en su conjunto, con lo cual las desigualdades políticas y económicas están siendo percibidas como diferencias culturales.

Žižek manifiesta que solo la cultura del tolerante puede considerarse como verdaderamente universal; sin embargo, esa tolerancia, que es una actitud políticamente correcta, resulta tener doble faz, la de la intolerancia y la represión en la medida en la que pide al Otro que siga atado a su identidad particular, permaneciendo en el lugar que le corresponde, en el lugar que le ha sido asignado socialmente para no perturbar el status quo. Ahora bien, el fenómeno que opera entre la denominada universalidad global y la universalidad concreta encuentra su respuesta a partir de la elaboración que Hegel hizo frente a la paradoja moderna de la individualización, a través de la identificación secundaria que se explica de la siguiente manera: el sujeto en su origen está inmerso en la forma de vida particular en la cual nació, en su comunidad orgánica primordial (su familia y su comunidad local); el único modo de romper los vínculos con ella y afirmarse como individuo autónomo es reconocer la sustancia de su ser en otra comunidad secundaria que se caracteriza por la actividad de sujetos libres e independientes. De esta manera se dibuja la dicotomía entre comunidad local y comunidad nacional -Nación-. En este tránsito de una comunidad a otra, las identificaciones primarias se transforman y empiezan a funcionar como la forma en que se manifiesta la identificación secundaria universal y se establece una analogía que permite afirmar que precisamente por ser un buen miembro de familia ese ser humano contribuye al funcionamiento correcto del Estado-Nación (Jameson, 1998, págs. 164-165).

Con lo antes expuesto, resulta posible afirmar que globalización no es universalidad, toda vez que globalización se refiere a la homo- 
genización planetaria del orden económico denominado capitalismo, es decir, es la hegemonización de los contenidos particulares del capitalismo bajo una pretendida universalidad. Se entiende así que la forma ideal de la ideología del capitalismo global sea el multiculturalismo, al que puede definirse como la forma de tratar a cada cultura local igual a la forma como el colonizador trata al pueblo colonizado: "como nativos cuya mayoría debe ser estudiada y respetada cuidadosamente". ${ }^{\prime}$

Y es precisamente aquí donde cualquier encuentro con el otro se convierte en un escenario de disputa que puede ser encontrado en las dinámicas de comportamiento electoral, y en el caso particular colombiano, causal de la construcción del denominado "castrochavismo". En el mundo multicultural se aprecia un conjunto de actitudes liberales hacia el Otro que, al mismo tiempo, revelan una paradoja, pues de un lado existe un respeto profundo por su alteridad y, por el otro, un temor frente al riesgo de hostigamiento, de intrusión, de invasión. En el caso colombiano, tras diferentes eslóganes y publicidad se ha invitado a respetar al venezolano migrante, con diferentes programas televisivos ha quedado la idea de que el gobierno venezolano no es igual al gobierno de Colombia, que este sí es bueno, y que la intensión de ayuda al otro es una característica de los colombianos. Se han convertido, pues, en seres caritativos y compasivos con la tragedia del país bolivariano. Sin embargo, al mismo tiempo se le juzga de ser ladrón, de ser asesino y de no pertenecer al territorio. Los diarios y la televisión también alimentan esta percepción al poner en sus titulares acciones violentas generadas por venezolanos con título propio.

Hay un temor, pues, a que este fenómeno causa del comunismo, según los políticos de derecha, se repita en el país, y a volverse los co-

9 “La relación entre el colonialismo imperialista tradicional y la auto colonización capitalista global es exactamente la misma que la relación entre el imperialismo cultural occidental y el multiculturalismo: de la misma forma que en el capitalismo global existe la paradoja de la colonización sin la metrópolis colonizante de tipo Estado-Nación, en el multiculturalismo existe una distancia eurocentrista condescendiente y/o respetuosa para con las culturas locales, sin echar raíces en ninguna cultura en particular"' (Jameson, 1998, pág. 172). 
lombianos en los venezolanos de hoy. Es así como se constituye esta paradoja en que existe la disposición de respetar al Otro siempre que su presencia no sea tan próxima.

Para esta lectura, se encuentra en esta paradoja la explicación para el nacimiento de los nacionalismos, pues su sentido es excluir los valores de los otros que son ajenos a la propia comunidad, con lo cual es válido afirmar que en los nacionalismos hay goce en la negación del goce del otro, quien vibra con valores culturales diferentes. Precisamente acerca de los nacionalismos Žižek considera que la denominada cosa nacional existe si los miembros de una comunidad creen que existe, por lo tanto, el nacionalismo es un efecto de la creencia por parte de una comunidad ideológica de creyentes y no una cosa en sí misma.

En este orden, la tolerancia que se aprecia en el multiculturalismo actual construye fronteras entre ciudadanos en un mismo territorio: los normales, los colombianos y los otros, los venezolanos, los de los grupos minoritarios, que deben sucumbir a las redes de la identidad cultural prevaleciente por ser ellos los de la condición diferente, generando un proceso de segregación al interior de la sociedad. En este sentido, en el mundo capitalista multicultural, el otro ${ }^{10}$ no es reconocido en su plenitud pues está sujeto a las condiciones de la identidad cultural de la mayoría que lo limita a partir de las creencias religiosas, las costumbres, las prácticas sociales, las preferencias sexuales que caracterizan a la mayoría social. En ese sentido, se trata, en palabras de Santiago Castro-Gómez, "de una tolerancia, que respeta al otro, pero manteniéndolo a una distancia segura" (2015, pág. 164).

10 Lo que se denomina el pensamiento de "los Otros" no es tal, pues es el pensamiento de una parte del mundo a la cual se le ha negado su lugar constitutivo en el todo y se le han rehusado sus elementos de identificación, como sus clases, etnias, culturas y géneros dominantes, transformándola en "la parte que no tiene parte" de la que habla Jacques Rancière. Por ello, cuando se dice "los Otros" se habla no tanto la lengua de los vencidos como una lengua vencida, se admite una calificación asignada por la parte-amo bajo una pretendida comprensión tolerante (Grüner, 2002, pág. 48). 
El otro, a su vez, representa la condición de su goce, bajo el entendimiento que el goce del sujeto está dado a partir de la negación del goce del otro, puesto que el otro no puede ser realmente él, por lo menos no bajo la condición de que su presencia resulte intrusiva. Lo que se desprecia del otro es lo excesivo de su goce, la aparente plenitud de su satisfacción por el hecho de que la imagen que proyecta el otro sea testimonio de una vida colmada, así resulta que esa manera de goce es intolerable por su proximidad.

En esta línea argumentativa, el sujeto se ve afectado a causa de otro que muestra su completud, es decir, que mientras el sujeto no consigue encontrar un objeto que colme su deseo se mira en otro que se siente colmado, completo, pleno -en apariencia-, y cuyo goce se convierte en una afrenta para su vacío. Esta relación se establece en el marco de un lazo social que desde la óptica lacaniana genera envidia, más no por el objeto en sí mismo, sino por el goce que aparentemente le aporta al otro sujeto:

Más que de un deseo de poseer, se trata de odio hacia el otro en tanto parece estar gozando. (Braunstein, 2014) Odio que recae en el goce del otro, su celo goce (jalouissance), según el neologismo forjado por Lacan. Celos del goce supuesto en el otro, pero también goce por estos celos, cuya dimensión mortífera presentimos, dimensión a-social. Puede haber un odio tenaz que toma por blanco al otro, en tanto y en cuanto éste gozaría, y ese odio es en sí mismo goce al cual el sujeto no quiere renunciar. La recriminación contra el prójimo que goza de algo de lo que el sujeto se siente privado, entraña un placer en exceso que parece nutrirse de su propia insatisfacción. (Chaumon, 2005, pág. 63).

En consecuencia, a partir de esta argumentación, la idea de que existan valores cultures que se consideran por una comunidad como propios hace que la presencia de otras comunidades culturales genere un sentimiento de amenaza. Es este sentimiento el que da lugar al nacionalismo que busca eliminar a los otros con el propósito reconstituir la "estabilidad" que supuestamente había. No obstante, considera que el nacionalismo, "la cosa nacional", existe siempre y cuando los miembros de una comunidad ideológica de creyentes crean que existe. 
En este orden, este nacionalismo exacerbado llevó a que en Colombia ganara Iván Duque, por su política castrochavista que concuerda con un momento histórico para el país que es del acuerdo de paz (CNMH, 2013). De la mano de una arremetida en su imagen sin parangón, iba subiendo el sentimiento de desconfianza frente al proceso de paz y el miedo al castrochavismo, o lo que es igual, a convertirse en una nueva Venezuela. Como lo muestra INVAMER, una de las casas encuestadoras más acertadas en las elecciones de 2018, hubo diferentes variables que explican el voto a Duque, entre las que se encuentran un incremento en la intención de voto exponencial de septiembre de 2017 a marzo de 2018, del $10.6 \%$ al $48.0 \%$; el incremento de una imagen favorable del $8.2 \%$ al $62.4 \%$ en el mismo lapso; una imagen desfavorable del proceso de paz, del $45.1 \%$ en diciembre del 2018; y una imagen desfavorable de Las FARC del $86.6 \%$. A su vez, se percibió que el proceso de paz iba por un mal camino, con un $62.4 \%$, y el 50 \% de la población consideró que Colombia podría estar, en un futuro, en la misma situación que Venezuela (INVAMER, 2018).

Problemas estructurales y que afectan a la población, como la guerra por más de 60 años (Duncan, 2006), la pobreza y la falta de acceso a mínimos vitales, estuvieron por debajo de este discurso, un discurso que permitió reflejar cómo es que, a partir de la simbolización de la realidad tras el discurso del otro, es que se genera una concepción del mundo en que se vive y la que se defiende. Así, a partir de la rememoración de experiencias pasadas e información recibida del proceso gubernamental venezolano, Iván Duque estableció un discurso que, como lo diría Laclau (2004), logró una lógica equivalencia, desde el que se unieron varios grupos sociales sin importar sus características, con la intensión de contrarrestar la amenaza del castrochavismo y poner a salvo su concepción del mundo.

Es decir, racionalmente eligieron un candidato que eliminaría las amenazas a su forma de ver el mundo. Y es que, en efecto, las personas sintieron miedo a que el país se volviera como Venezuela, rabia a que se implementara el acuerdo de paz y eso llevara a establecer políticas de izquierda, beneficios para excombatientes $y$, a su vez, simpatía hacia el candidato que postulaba una lucha contra aquello generador de miedo. No obstante, el voto emocional del que habla la 
inteligencia afectiva es un voto racional que se centra en los afectos de los electores, en aquello que pueden nombrar y conscientemente decir que sienten a través de un proceso cognitivo. De esta manera, esta decisión que, en realidad, es consciente, encubre que el verdadero voto emocional es un voto irracional, y que es posible gracias a la apariencia de racionalidad al complementarse con el reconocimiento en el otro. Por esta razón, el individuo siempre está intentando afirmar su decisión, tanto con otros como con información en general que permita cruzar lo que sabemos con lo que sentimos, pues sin esta afirmación el individuo no concibe su pensamiento ni estará tranquilo con su forma de ser en el mundo.

En esta lógica, sin desconocer que las estadísticas para explicar la tendencia al voto pueden dar cuenta de variables explicativas emocionales, hay casos en que las personas eligen gobiernos que van en conta de su propio bienestar -sobre todo aquellos autoritarios de derecha y derecha radical-, y en los que la emotividad "inconsciente" no pueden dar cuenta de dicho comportamiento.

Quizá por ellos, la privación del goce para los migrantes, los independistas, los revolucionarios, los negros, los indígenas, los homosexuales, los excombatientes, los sudamericanos, los africanos, los musulmanes, los ambientalistas, los animalistas, los venezolanos, en este caso, etc., puede también determinar el voto de una forma inconsciente, pues aquello me hace gozar. Si los gobiernos de derecha son ellos mismos quienes sacan a flote estos temas y ponen en el discurso público la negación de goce, se genera una suerte de tranquilidad para hablar, decir lo que se siente y negar incluso de manera pragmática el goce del otro; los gobiernos autoritarios permiten que esta negación pase por un asunto real, al otro se ataca, se insulta, se ignora y se mata, aunque el placer no está en matarlo sino en negarle su goce y continuar la búsqueda insaciable de alcanzar el propio sin poder lograrlo; por ello muchos gobiernos dan la opción de que estas personas cambien, se conviertan o paguen por sus penas, pero sin eliminarlos, porque al matarlos, matan también el goce. ${ }^{11}$

11 Para ampliar el tema, revisar Žižek, S. (1994). Goza tu sintoma. Jacques Lacan dentro y fuera de Hollywood. Nueva Visión. 
En este sentido, se puede decir que el castrochavismo fue este discurso que movilizó lo Real, que movilizó el voto de odio hacia el otro, es decir, que movilizó el goce. Ivan Duque, Álvaro Uribe Vélez, y varios políticos del Centro Democrático ${ }^{12}$, incitaron sentimientos nacionalistas y xenófobos para llegar al poder, poniendo el goce de colombianos a funcionar y mostrando el síntoma del multiculturalismo capitalista.

Ahora bien, la movilización de ese goce, traspasado por la negación del goce del otro, en este caso del venezolano y su crisis, se puso como causa de los problemas de los colombianos, de aquellos que viven sin ser causantes de lo que pasa en Venezuela. No solo en este caso, en múltiples casos la causa de los problemas siempre es el otro, el migrante. Lo que no se evidencia es que detrás de esta excusa existe una real causa que son las consecuencias del sistema capitalista que per se es excluyente y generador de prácticas desiguales. Existe pues una relación entre el capitalismo multicultural y la exclusión social, que es difícil de evidenciar y que se suele mirar como causa del otro y no del sistema en general (Žižek, 2014).

Esta falacia multicultural se explica, según Žižek, porque la democracia es la manera políticamente correcta para incluir a los excluidos en el espacio sociopolítico bajo el sofisma de la igualdad, de que todos tienen las mismas oportunidades y derechos dentro de los Estados libres. (Žižek, 2011a). No obstante, cada quien lo tiene dentro de su Estado, el discurso global de los derechos humanos se limita a la hora de reclamarlos fuera del país de origen, pareciendo, por ello, un cierto acto de "robo" de los derechos cuando los migrantes los pueden ejercer dentro del territorio.

12 El Centro Democrático es un partido político colombiano fundado en 2013 por el expresidente Álvaro Uribe Vélez. El partido se define a sí mismo como un partido de ciudadanos de diversos orígenes políticos basado en cinco pilares: seguridad democrática, confianza inversionista, cohesión social, estado austero descentralizado y diálogo popular. Su ideología es de derecha. 


\section{Conclusiones}

Los estudios sobre el comportamiento electoral han prestado atención en los últimos años al papel de las emociones en la política. En ese sentido, utilizando técnicas como encuestas con preguntas enfocadas en medir variables emocionales han estudiado cómo influyen estas en la elección o no de un candidato político. Sin embargo, en no pocos casos las encuestas fallan y se presentan fenómenos inesperados para los ciencistas políticos y estudiosos del escenario electoral. Una de las causas de dichos sucesos tiene que ver con que estos estudios se quedan en el ámbito contextual y racional del individuo, es decir, en el escenario óntico. Así, la pregunta de qué emociones siente un ciudadano tiene una tendencia a ser respondida con claridad por las encuestas. Sin embargo, la pregunta de por qué se sienten esas emociones, es sin duda una tarea que requiere un análisis interdisciplinario.

Desde la filosofía política, específicamente el posmarxismo, se sostiene que las razones que generan emociones se deben estudiar en el escenario ontológico, es decir, en la constitución del sujeto, y a partir de ahí entender cómo los discursos políticos movilizan lo que se es. En este orden, y teniendo presente que en el año 2018 fue electo como presidente el señor Iván Duque -a partir de un discurso emocional denominado castrochavismo, donde los principales protagonistas fueron Venezuela, los venezolanos y el temor de los colombianos de convertirse en el país vecino por su crisis económica y migratoria actual-, podría explicarse este fenómeno del voto acudiendo a los presupuestos posmarxistas a fin de complementar las lecturas más politológicas.

Tomando como base la teoría propuesta por Slavoj Žižek, el goce del sujeto se circunscribe en un deseo de negarle el goce al otro, y desde ahí se puede entender por qué son rechazados los venezolanos en una construcción de cosa nacional generada por discursos políticos xenófobos y excluyentes. El discurso de Iván Duque, el castrochavismo, puede comprenderse desde esa dinámica: emociones de odio al otro, al diferente, al que no debería estar aquí sino allá, en su crisis, 
crisis que además está todo el tiempo latente en Colombia a causa de los políticos pro paz, que se han asimilado a pro FARC ${ }^{13}$.

\section{Referencias}

Braunstein, N. (2014). El goce, un concepto lacaniano. Siglo Veintiuno Editores. Castro, E. (2004). El vocabulario de Michel Foucault: un recorrido alfabético por los temas, conceptos y autores. Prometeo-Universidad Nacional de Quilmes.

Castro-Gómez, S. (2015). Revoluciones sin sujeto. Slavoj Žižek y la crítica del historicismo posmoderno. Ediciones Akal.

Centro de Nacional de Memoria Histórica (CNMH). (2013). Basta Ya. Memorias de guerra y dignidad. Imprenta Nacional.

Chaumon, F. (2005). La ley, el sujeto y el goce. Lacan y el campo jurídico. Nueva Visión.

Downs, A. (1975). An Economic Theory of Democracy. Harper \& Row.

Duncan, G. (2006). Los señores de la guerra. Planeta.

Grünner, E. (2002). El fin de las pequeñas historias. Paidós.

Investigación y Asesoría de Mercado (INVAMER). (2018). https://www.invamer.com.co/es/

Jameson, F. y Žižek, S. (1998). Estudios culturales. Reflexiones sobre el multiculturalismo. Paidós.

Lacan, J. (1957/1958). El seminario, libro 5: Las formaciones del inconsciente. Nueva Visión.

Lacan, J. (1960). Subversión del sujeto y dialéctica del deseo en el inconsciente freudiano. [1985]. En Escritos 2, (págs. 773-807). Siglo Veintiuno Editores.

Lacan, J. (1975). Escritos 2. Siglo Veintiuno Editores.

Lacan, J.(1964). El seminario, libro II: Los cuatro conceptos fundamentales del psicoanálisis. Paidós.

Lacan, J. (2006/1975-1976). El seminario, libro 23: El sinthome. Paidós,

Marcus, G., Neuman, W, R. \& Mackuen, M. (2000). Affective Intelligence and Political Judgment. The University of Chicago Press.

Molano, A. (2016). A lomo de mula: viajes al corazón de las FARC. Aguilar.

Rodríguez de Dios, O. (2008). La ontología política de Slavoj Žižek. Escatología $y$ sintoma. UNED.

13 Fuerza Armadas Revolucionaria de Colombia, nombre que tuvo la guerrilla colombiana de mayor data hasta el año 2012, hoy denominado partido Comunes. 
Žižek, S. (1998). Porque no sabe lo que hacen. El goce como factor político. Paidós. Žižek, S. (2001). El espinoso sujeto. El centro ausente de la ontología política. Paidós.

Žižek. (2003a). El sublime objeto de la ideología. Siglo Veintiuno Editores.

Žižek, S. (2003b). Ideología, un mapa de la cuestión. Fondo de Cultura Económica.

Žižek, S. (2003c). Porque no saben lo que hacen, el goce como factor político ( $2^{\mathrm{a}} \mathrm{ed}$.). Paidós.

Žižek, S. (2006). Arriesgar lo imposible. Conversaciones con Glyn Daly. Trotta.

Žižek, S. (2008a). Cómo leer a Lacan. Paidós.

Žižek, S. (2008b). En defensa de la intolerancia. Sequitur.

Žižek, S. (2011a). En defensa de las causas perdidas. Akal.

Žižek, S. (2011b). Primero como tragedia, después como farsa. Akal.

Žižek, S. (2014). Pedir lo imposible. Akal. 